\title{
CD8-Positive T-Lymphocyte
}

National Cancer Institute

\section{Source}

National Cancer Institute. CD8-Positive T-Lymphocyte. NCI Thesaurus. Code C12542.

A subset of T-lymphocytes that express the CD8 surface glycoprotein and bind epitopes that are part of class I histocompatibility molecules. CD8-positive T-lymphocytes include cytotoxic T-lymphocytes and CD8+ suppressor T-lymphocytes. 